\title{
Effects of reducing or modifying dietary fat on CVD: a systematic review and meta-analysis of randomised controlled trials
}

\author{
L. Hooper ${ }^{1}$, C. D. Summerbell ${ }^{2}$, R. L. Thompson ${ }^{3}$, D. Sills ${ }^{1}$, F. Roberts ${ }^{1}$, H. J. Moore ${ }^{2}$ \\ and G. Davey Smith ${ }^{4}$ \\ ${ }^{1}$ Department of Nutrition, University of East Anglia, Norwich NR4 7TJ, UK, ${ }^{2}$ School of Medicine and Health, \\ Durham University, Stockton-on-Tees TS17 6BH, UK, ${ }^{3}$ World Cancer Research Fund International, 22 Bedford Square, \\ London WCIB 3HH, UK and ${ }^{4}$ Department of Social Medicine, University of Bristol, Bristol BS8 2PS, UK
}

The effect of reduction of energy from dietary fats has differing effects on cardiovascular risk factors (such as serum cholesterol), but its effects on CVD is less clear. Our previous systematic review of randomised controlled trials, published 10 years ago, and a recent systematic review of the epidemiological evidence, both suggested little effect of dietary fat reduction on CVD ${ }^{(1,2)}$. The aim of this systematic review was to assess the up-to-date evidence of the effect of reduction and/or modification of dietary fats on mortality, cardiovascular mortality, cardiovascular morbidity in randomised clinical trials of at least 6 months duration.

The Cochrane Library, MEDLINE and EMBASE, were searched through June 2010. References of included studies and reviews were also checked. Included trials fulfilled the following criteria: (1) randomised with appropriate control group, (2) intention to reduce (or reduce and modify) fat intake (excluding exclusively omega-3 fat interventions), (3) not multi factorial, (4) adult human subjects with or without CVD, (5) intervention at least 6 months, (6) mortality or cardiovascular morbidity data available. Assessment of inclusion was duplicated, disagreement resolved by discussion or a third party. Participant numbers experiencing health outcomes in each arm were extracted independently in duplicate and random effects meta-analyses, meta-regression, sub-grouping, sensitivity analyses and funnel plots were performed.

This updated review suggested that reducing saturated fat by reducing and/or modifying dietary fat reduced the risk of cardiovascular events by $14 \%$ (RR 0.86 , 95\% CI $0.77-0.96$, twenty-four comparisons, 65508 participants of whom $7 \%$ had a cardiovascular event, $\mathrm{I}^{2} 50 \%$ ). Subgrouping suggested that this reduction in cardiovascular events was seen in studies of fat modification (not reduction), of at least 2 years duration, in studies of men (not of women), and in those with moderate or high cardiovascular risk at baseline. There were no clear effects of dietary fat changes on total mortality (RR 0.98, 95\% CI 0.93-1.04, 71790 participants) or cardiovascular mortality (RR 0.94, 95\% CI 0.85-1.04, 65978 participants). This did not alter with sub-grouping or sensitivity analysis.

Few studies compared reduced with modified fat diets, so direct comparison was not possible.

The findings are suggestive of a small but potentially important reduction in cardiovascular risk on modification of dietary fat, but not reduction of total fat, in longer trials. Lifestyle advice to all those at risk of CVD and to lower risk population groups, should continue to include permanent reduction of dietary saturated fat and partial replacement by unsaturates. The ideal type of unsaturated fat is unclear.

This systematic review is due to be published as an updated Cochrane Review (CD002137).

1. Hooper L, Summerbell CD, Higgins JPT et al. (2001) BMJ 322, 757-763.

2. Siri-Tarino PW, Sun Q, Hu FB et al. (2010) Am J Clin Nutr 91, 535-546. 